\title{
Empowering OpenMP with Automatically Generated Hardware
}

\author{
Artur Podobas and Mats Brorsson \\ School of Information and Communication Technology \\ Royal Institute of Technology \\ Kistagången 16,16440 Stockholm, Sweden \\ \{podobas,matsbror\}@kth.se
}

\begin{abstract}
OpenMP enables productive software development that targets shared-memory general purpose systems. However, OpenMP compilers today have little support for future heterogeneous systems - systems that will more than likely contain Field Programmable Gate Arrays (FPGAs) to compensate for the lack of parallelism available in general purpose systems.

We have designed a high-level synthesis flow that automatically generates parallel hardware from unmodified OpenMP programs. The generated hardware is composed of accelerators tailored to act as hardware instances of the OpenMP task primitive. We drive decision making of complex details within accelerators through a constraint-programming model, minimizing the expected input from the (often) hardware-oblivious software developer.

We evaluate our system and compare them to two state of the art architectures - the Xeon PHI and the AMD Opteron - where we find our accelerators to perform on par with the two ASIC processors.
\end{abstract}

\section{INTRODUCTION}

OpenMP is a standard programming model used to exploit parallelism within an application. It allows programmers to annotate source code with compiler directives, indicating where parallelism can be exploited. An OpenMP compiler can transform these directives to parallel, often high-performant, general-purpose processor code.

Currently OpenMP is a model to write software for use in general purpose computer architectures. The work presented in this paper enables OpenMP to be a model for writing software and hardware with little extra effort. The hardware generated can be synthesized and executed on field-programmable gate arrays (FPGAs).

The trend to overcome failing Dennard scaling is to exploit heterogeneity in computation through accelerators - today, acceleration is obtained through GPUs but it is likely that the far more customizable FPGAs will complement that role. In addition, the industry has also seen value in this approach and architectures such as Xilinx ZYNQ and the Intel/Altera platforms are emerging.

There are several reasons why enabling OpenMP-driven high-level synthesis (HLS) is worthwhile. Custom built FPGA hardware is more efficient than general purpose components [10], [7]. Power-consumption - a first-class constraint

This work has been funded by the Artemis PaPP project number 295440
- can also be reduced compared to CPUs or GPUs [11], [8]. FPGAs can be configured to have far more parallel throughput than their general purpose counterparts. Granted, FPGAs (or rather, their development boards) arguable costs more than any general purpose CPU or GPU; however, it is likely that the growing interest of vendors will make FPGAs more readily available in the near future (for example Intel QuickAssist for FPGAs or Xilinx ZYNQ).

No matter what benefits FPGAs brings, building custom hardware by hand is time consume and prone to error. Moreover, there exists one hardware engineer for every ten software engineers [12], justifying the need for ease-to-use tools for hardware generation. Even existing HLS solutions needlessly expose too many options to the software developer.

This paper uses BŁYSK [19] - our prototype OpenMP framework, where we added a novel strategy for sharing resources across hardware instances of OpenMP tasks called hyper-tasks. We call these shared resources regional components and show how they can be used to increase performance through parallelism in hardware that is automatically generated. These hyper-tasks require little-to-no input from the developer - the application is analyzed and a constraint-based solver is used to decide the fine details other tools expects the user to supply.

Our framework accepts OpenMP annotated C-code and transforms it into a System-on-Chip composed of a softcore and a number of hardware accelerators that are fully dedicated to perform OpenMP task-parallel applications as fast as possible. To our best knowledge this is the first time accelerators that utilize both MIMD and SIMD parallelism are automatically generated and modelled with componentsharing between asynchronous parallel computational units, closely tied to the parallel model. We show how both the hyper-tasks and the regional component can be implemented, how to decide on their usage and what constraints are imposed on the hardware and the compiler.

We evaluate hyper-tasks and their scalability and show that it is worthwhile sharing resources to gain performance using FPGAs. We also put our generated systems in perspective by comparing them to existing OpenMP-capable general purpose hardware. 


\section{RELATED WORK}

Overall, high-level synthesis of parallel workloads can be divided into two categories: data-path and state-machine based.

Data-path based HLS involves creating a large data-path, into which data is being push into. The data-path is highly pipelined allowing a high degree of parallelism and throughput. Often the parallel concept of threads is used to decide what data to be fetched into the data-path. Examples of datapath parallel HLS today is for example the work of Halstead et al. [9], Altera's OpenCL [5] or FCUDA [17]. The benefits of data-path based HLS is that a high-degree of throughput can be obtained. However, data-path construction typically limits the amount of control-flow that can exist in the kernel. Controlflow statements are often predicated and both paths (true and false) execute in parallel.

State-machine based HLS is more generic than the data-path based version. Here, each instruction is mapped into a finitestate machine that governs how data flows through the units and state is maintained globally. There are fewer limitations concerning control-flow but comes at the cost of performance. Examples of state-machine based parallel HLS-tools include the work by Leow et al. [13] and Nowak et al. [16] (albeit microcoded).

Our approach combines both data-path and state-machine execution and is similar to LegUP [3]. Instructions are mapped into a state-machine while basic blocks without control-flow are decomposed into data-paths. Additionally, unlike existing work, our work promotes sharing of expensive resources. We formally model the problem of sharing as a constraint optimization problem unlike related work that uses heuristics [3], [4]. Our work also allows resource sharing across multiple hardware instances while related work primarily considers reusage of components within one hardware instances.

Cabrera [2] et al.studied FPGA co-acceleration in OpenMP from a run-time system perspective using OmpSs. Their work primarily concerned quantifying overheads in using FPGAs from the runtime-system's perspective (such as DMA transfers and reprogramming the FPGA) - no high-level synthesis was performed and existing IP cores were used. Later, Filgueras et al.[6] continued to work by introducing directives into their Mercurium compiler, which in turn were used to synthesize VHDL code using Xilinx Vivado. Our work differs in that our tool both source-to-source compiles and performs the highlevel synthesis of the OpenMP application.

DURASE [14] is a system for automatically generating hardware for certain portions of a serial applications code. As with the present work, DURASE model the problem using constraint programming. However, DURASE is limited to serial application and only focuses on matching tree's in the intermediate code to decide what portions should be accelerated - they are oblivious to the problem of sharing resources across several asynchronous processing units, which is what the present paper tackles.

\section{OPENMP-DRIVEN HLS OVERVIEW}

The steps performed by our framework are seen in Figure 1:a.
The user provide an OpenMP annotated source code, which is the basis for the custom system-on-chip that our framework generates. We focus on exploiting parallelism using asynchronous tasks (\#pragma omp task) with (OpenMP 4.0) or without (OpenMP 3.0) data-flow information. Every detected OpenMP task will be converted into what we call a hyper-task - a custom hardware unit that perform the work within a particular task. Several hyper-tasks are clustered together for resource efficiency and are then called an accelerator (Figure 1:b). Once accelerators have been created, they are bundled together with a NiosII soft-core and a systemon-chip is created. The system-on-chip is very similar to the IBM Cell processor, where the NiosII acts as a master towards the OpenMP accelerators. Knowing the layout of the SoC, the compiler now can proceed to translate remaining parts of the source-code to exploit the newly generated SoC. All detected OpenMP constructs related to the tasks are source-to-source translated into using the generated accelerators. The output is an Altera QSYS hardware description file and an associated $\mathrm{C}$ file for the NiosII processor.

\section{ACCELERATORS AND HYPER-TASKS}

The hyper-tasks generated by our method are composed of a number of primitive components (such as adders or multipliers). Each hardware component is associated with a component declaration (ports), number of pipeline stages and a tree-match it represents in the intermediate dependencygraph. We refer to components that are shared within one hyper-task as private. A private component can be promoted to become shared across hyper-tasks and is then called a regional component (section IV-A).

A private hardware component can be used by several intermediate instructions (within one hyper-task) as long as they start at different times. When a component is used by several instructions, multiplexers will be added to choose inputs into the component. Components that are private require the scheduled instructions to capture the results according to the component's latency (the result is unbuffered).

We support arbitrary wide vectorization instructions that can be used from within the $\mathrm{C}$ source code. The vector components are automatically formed by linking several smaller components together. Exploiting vectorization requires the programmer to use certain pre-defined builtin-functions (conceptually similar to [21]).

\section{A. Regional Components}

Original to this paper are components that can be shared between hyper-tasks. We call them regional components. Any component, from the smallest of adders to the largest of crossproducts can be a regional component. Figure 2 illustrates the internals of a regional component.

Unlike private components, regional components are extended with logic to support sharing. An arbiter is integrated into the component. The arbiter will choose one of the many possible hyper-tasks (HTs) that are requesting to use the regional component. We chose a fixed-priority based arbitration scheme. 
a) Overall Compilation Flow

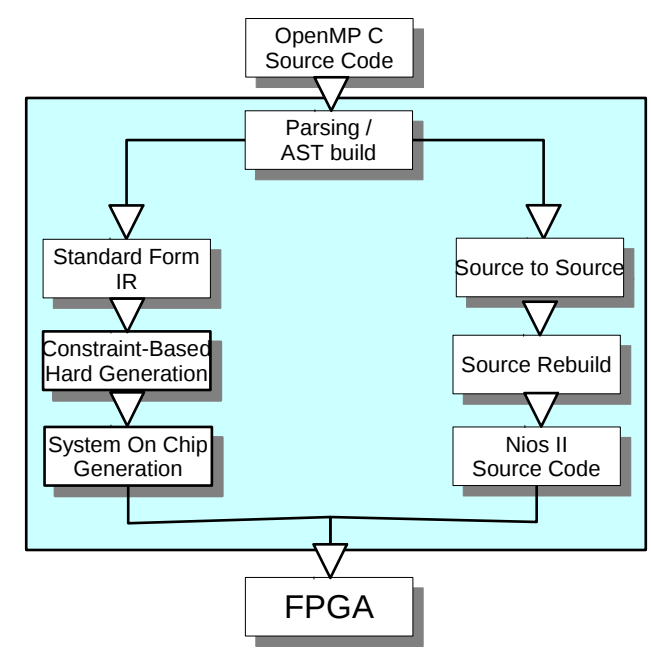

b) An OpenMP Accelerator

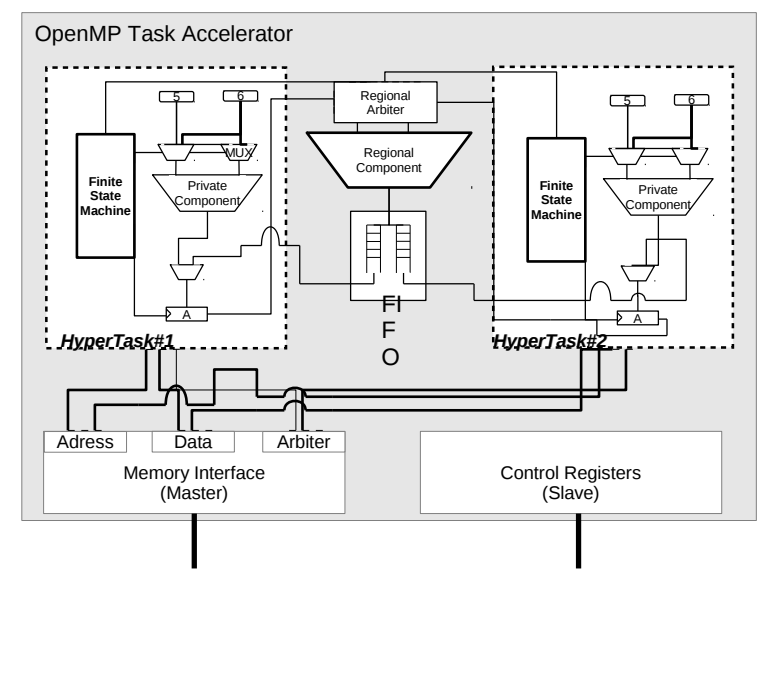

Fig. 1: Overview over the steps and component involved in transforming an OpenMP-extended C source code into a specialized System-on-Chip (a) and an abstract overview over one OpenMP accelerator containing two hyper-tasks with both regional and private components (b).

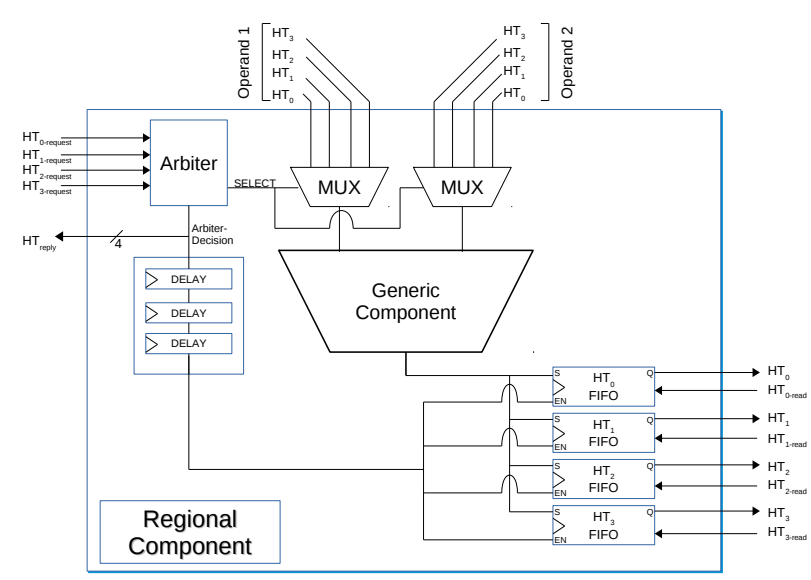

Fig. 2: Promotion of a generic (local) component to a regionalcomponent by extending it with arbitration details.

The fixed-priority arbitration assumes that a number of requesters $H T_{1}, H T_{2}, \ldots, H T_{n}$ attempt to use the regional component. The fixed-priority arbitration will prioritize the lowest ranked requesters, meaning that $H T_{1}$ will have uncontested access to the component, $H T_{2}$ will acquire it only if $H T_{1}$ does not request it and so forth.

The arbiter's decision is a bit-vector, where each bit is sent to each of the hyper-tasks. The arbiter's decision is saved and pipelined alongside the computation and decides which of the FIFO buffers sitting at the end of the pipeline the result should be captured into. The latency of a regional component is always one clock cycle longer than its private counterpart because of the FIFO buffer.

\section{B. Generating Accelerators and Hypertasks}

The back-end of our compiler will generate an accelerator for the OpenMP-task given its intermediate representation code. The generated accelerators are memory-mapped, sitting on an Avalon [1] interconnect. They act as slaves towards the host processor (the NiosII) and as master towards other units (such as RAM). Communication between the Host and the Accelerator is performed through control-registers and include functionality such as associating a hyper-task with an address, assigning identifiers to each hyper-tasks or accelerators (which allows SPMD/SIMT execution or redundancy computing if desirable) and status registers for starting task execution.

1) Building the Accelerator: Building the accelerator involves mapping the intermediate representation into the hypertasks as well as deciding what resources to be shared within an accelerator. Intuitively, large components should be made regional in order to conserve FPGA resources, while small components should be made private to reduce multiplexer cost. This intuition does however come with problems. Depending on what operands are used for the components, it might still be worthwhile to make large components private. For example, a kernel that consists of a large number of duplicated fadd $\$ 1, \$ 2, \$ 3$ instructions can have the floating-point adder held private in each hypertask, reuse the adder continuously with minimal multiplexer pressure (since operands do change).

Another problem is the DSP-block pressure. FPGA resources can be divided into two types: ALMs (or LUTs) which is the internal block that logic operations are mapped to, and DSP which are hard-wired components used to assist for example multiplications. A kernel that is large on for example multiplications (which is an ALM friendly component because 
it uses DSP blocks) will therefore benefit from having the multiplication unit regional rather than private.

Transforming the intermediate representation into hardware is a known NP-hard problem. Unlike related work that use heuristics to solve above-mentioned problems, we solve it as an optimization problem through a constraint satisfactory problem (CSP). Constraint Programming is appealing: the model is formed and use a branch-and-bound engine to repeatedly search for a solution, adding constraints that monotonically improve the solution. The problem formulation was implemented through Gecode [20].

Our model uses the following variables:

\begin{tabular}{ll}
\hline Variable & Description \\
\hline$G \in \mathbb{Z}$ & Maximum number of resource of a given type \\
$n \in[1,16]$ & Number of Hyper-tasks per accelerator \\
$i \in I$ & An instruction \\
$R_{i} \in[-G,+G]$ & What resource instruction $i$ will use. \\
& Negative resources are regional while \\
& positive (including 0) are private \\
$S_{i} \in[0,+G]$ & Scheduled state of $i$ in the FSM \\
$C_{i} \in[0,1]$ & Commutative property of instruction $i$ \\
$Y_{i} \in[0,60]$ & Which component instruction $i$ uses \\
$O_{i_{j}} \in[0, G]$ & (we have 60 different components) \\
\hline
\end{tabular}

$I, O$ and $Y$ are known a-priori, obtained from the intermediate representation (the number of instructions, their type and which operands they use). Variable $G$ is bound through statistical-analysis a-priori to the computation. For the kernels we have used, $G$ is often bound to 4 and $O$ bound to the total number of different registers/constants used in program.

Our goal is to maximize the performance-metric $M$. The performance metric is calculated using an approximation of the number of instruction executed per cycle (IPC) linearly scaled by the number of hyper-tasks in the design over the area estimated for the design:

$$
M=\max \left(\frac{|I| * n}{t}\right)
$$

Estimating the cycle time to execute the kernel $(t)$ is calculated by summing up the last finishing instruction in each basic-block. Estimating the kernel execution time this way is often used. The metric $M$ is scaled to avoid the need for floating-point variables in the constrained model. The model has one hardwired constraint: the total number of DSP blocks used in the design must not exceed 256 (which is what our Stratix V FPGA contains).

2) Instruction scheduling constraints: Each instruction $i$ is scheduled on a state in the hyper-task's FSM, on a particular resource $R_{i}$ and uses a certain component group $Y_{i}$.

Two independent instructions $i$ and $j$ may not be scheduled on the same state should they use the same resource:

$$
\forall i, j \in I:\left(R_{i}=R_{j} \wedge Y_{i}=Y_{j}\right) \Longrightarrow S_{i} \neq S_{j}
$$

Similarly, an instruction $j$ which is dependent on an instruction $i$ must be scheduled after instruction $j$ have finished. An extra cycle is added should instruction $i$ be scheduled on a regional component:

$$
\forall i \in \operatorname{dep}(j): R_{i}<0 \Longrightarrow S_{i}+\operatorname{latency}\left(Y_{i}\right)+1<S_{j}
$$

$$
\forall i \in \operatorname{dep}(j): R_{i} \geq 0 \Longrightarrow S_{i}+\operatorname{latency}\left(Y_{i}\right)<S_{j}
$$

Two instructions $i$ and $j$, which both use a regional component, may neither be scheduled on the same state (due to arbitration requests) nor finish when the other one is awaiting arbitration decision:

$$
\begin{gathered}
\forall i, j \in I: R_{i}<0 \wedge R_{j}<0 \Longrightarrow \\
\left(S_{i} \neq S_{j}\right) \wedge\left(S_{i}+\text { latency }\left(Y_{i}\right) \neq S_{j}\right) \wedge\left(S_{j}+\text { latency }\left(Y_{j}\right) \neq S_{i}\right)
\end{gathered}
$$

An instruction $i$ which uses a regional resource may not be scheduled on a state where another instruction $j$ that uses a private component is in flight ${ }^{1}$

$$
\begin{gathered}
\forall i, j \in I: R_{i}<0 \wedge R_{j} \geq 0 \Longrightarrow \\
\left(S_{i} \leq S_{j}\right) \wedge\left(S_{i} \geq S_{j}+\text { latency }\left(Y_{j}\right)\right)
\end{gathered}
$$

3) Area constraints: Modelling the area is crucial to get a metric that reflects the hardware that is generated (and synthesized). We empirically derived individual multiplexer, components, arbiter and fifo area (ALM) and DSP cost through isolated synthesis. Isolated synthesis provides us with an upper-bound on the area consumption because there will always be ALMs that are not fully utilized - ALMs that could be packed with logic in a larger design. This can be seen in the area-evaluation for one of the benchmarks, where our estimated area is larger than that of the synthesized version for a number of solutions (Figure 3). While there is a large deviation between the synthesized- and the model-area, they correlate well, meaning that the performance metric we optimize over is accurately reflected in the generated hardware. We call the functions that estimates the area: cost(), cost_mux(), cost_fifo() and cost_arbiter().

The area for the accelerator is calculated from the area estimated by the regional components (including arbiters and FIFOs), the privatized components (multiplied with the number of hyper-tasks) and the total multiplexer area.

$$
\begin{gathered}
A=\sum \text { Regional }+n * \sum \text { Private }+ \text { Mux } \\
\text { Muxivate }_{\text {out }}+\text { Mut }
\end{gathered}
$$

The Regional and Private components are constrained primarily through channeling. A boolean array $B$ is created of size $n * 2 * G$ - large enough to hold all information regarding the potential resource usage for each of the instruction. A ' 1 ' in the row of this array indicate that a particular resource has been decided for the instruction with the same row index, modeled (through channeling constraints) as:

$$
\begin{aligned}
& \exists i \in I, \forall j \in R: R_{i}=j \Longleftrightarrow B\left(Y_{i}, R_{i}\right)=1 \\
& \forall j \in Y: \text { Regional }_{j}=\sum_{i=-G}^{i<0} B(j, i) * \operatorname{cost}\left(Y_{j}\right) \\
& \forall j \in Y: \text { Private }_{j}=\sum_{i=0}^{i<+G} B(j, i) * \operatorname{cost}\left(Y_{j}\right)
\end{aligned}
$$

Constraining the component's input multiplexers is done similarly, but with operands instead of component-types. We are calculating the number of unique operands used for a

\footnotetext{
${ }^{1}$ Private instruction always capture the value according to their components latency. Any stalls (by waiting for arbitration) will make the private instruction capture an incorrect value.
} 


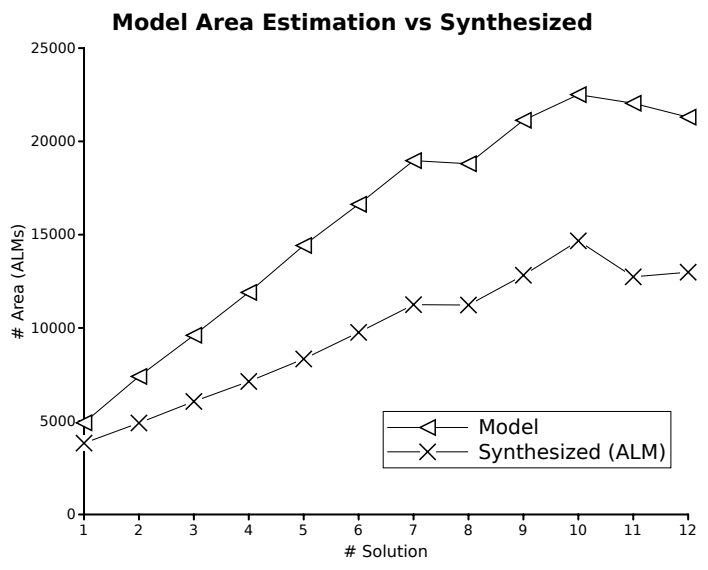

Fig. 3: Comparison between our area-model and the area resulting from synthesis of the hardware using Altera Quartus II.

particular resource $R$. If the same operand is used for a particular resource, then our backend will not duplicate the input but rather re-use an input.

A function, count $(y, r, p)$ is used, which calculates the number of unique operands used for a particular component $(y \in Y)$ and its resource $(r \in R)$ for one of its input ports (p). The total multiplexer cost is calculated as:

$$
\begin{aligned}
\text { Mux }_{\text {private }}= & \sum_{i=0}^{i<60} \sum_{j=0}^{j<G} \sum_{k=0}^{k<\operatorname{ports}\left(Y_{i}\right)} \operatorname{cost}_{\text {mux }}(\operatorname{count}(i, j, k)) * n \\
\text { Mux }_{\text {regional }}= & \sum_{i=0}^{i<60} \sum_{j=0}^{j<G} \sum_{k=0} \operatorname{cost}_{\text {mux }}(\operatorname{count}(i, j, k))+ \\
& \operatorname{cost}_{\text {arbiter }}(n)+\operatorname{cost}_{\text {fifo }}(n)
\end{aligned}
$$

Constraining the multiplexer area for the output $\left(M u x_{\text {out }}\right.$ (hut $)$ of operations is done similarly as for the input, albeit now the matrix count () is defined on the output operands rather than on the component/operand pair.

We model commutative properties of operations and operands and the model can chose to switch operands to potentially reduce the area (should the input already exist on one of the multiplexer to the component):

$$
\forall i \in I: C_{i}=1 \Longrightarrow \text { switch_operand }\left(O_{i_{1}}, O_{i_{2}}\right)
$$

\section{System-Generation}

We compose the entire system-on-chip once the accelerators have been described. We use the Avalon interconnect as a medium for communication. The programmer can choose how many accelerators will be mapped onto the Avalon interconnect. Each mapped accelerator will have its memory-mapped address recorded for further use by the compiler. Aside from the accelerators, a timer, block RAM holding instructions and data and a NiosII f-type processor will be mapped. The output will be given as a system (QSYS) description, with which existing Altera tools can be used to synthesize the design down to hardware.

\section{Source-to-Source Translation}

Aside from generating the system, our compiler needs to modify the original source-code so that it is capable of exploiting the auto-generated accelerators. Parts of this flow is illustrated in Figure 4 and involves transforming the two task-parallel constructs in OpenMP: omp task, omp taskwait.

In our case, the work-sharing construct omp parallel/single deviates from the OpenMP specification since it initializes the system for parallel execution but the accelerators do not execute the parallel region- instead they sit idle and wait for work to be scheduled to them.

Booting accelerators (Figure 4:b) requires knowledge of where those accelerators are memory-mapped in the systemgeneration step (see section IV-C). The boot-up sequence allocates memory holding the arguments to a task for each hyper-task in an accelerator and sets the accelerator's device_address to point into this shared memory position (Figure 4:c).

Creating and exposing a task involves translating the OpenMP directive so that a structure holding the task's arguments is allocated. The structure is initialized accordingly to the task's function arguments (Figure 4:b). Calling the submit_try_run () function (Figure 4:d) will finally submit the task to the run-time system. submit_try_run () is automatically generated and attempts to find an idle hypertask to schedule the work on. The idle hyper-task returned is based on the scheduling policy. Scheduling a task onto an accelerator involves copying the arguments of the task into where the hyper-task is expected to find the arguments according to the previously set device_address. Execution starts by asserting the correct bit in the Start control register belonging to the accelerator where the hyper-task is. Should no hyper-task be found, the task is pushed onto a centralized queue, thus postponing the execution.

Finally, the omp taskwait function that synchronizes (blocks) execution until all previously spawned tasks have finished is translated into a_sync_all_tasks () function call (Figure 4:c). __sync_all_tasks () loops though all accelerators, scheduling tasks onto them until no more tasks exists.

\section{E. Runtime-System Scheduling Policy}

Our OpenMP runtime system's scheduling policy decides on which hyper-task in which accelerator an OpenMP task is exposed by the application. Scheduling policies in modern general-purpose systems are complex, taking architectural details such as caches and memory hierarchy into account. Fortunately, such complex thinking is needless for the systemon-chips that our framework generates.

The one architectural detail that we need to take into account when scheduling is the arbitration details that regulate access to regional components. It can be crucial for performance reasons to schedule tasks onto the OpenMP accelerator with the least amount of tasks currently running (the accelerator that is least loaded). The reason is illustrated in Figure 5. Here, an 


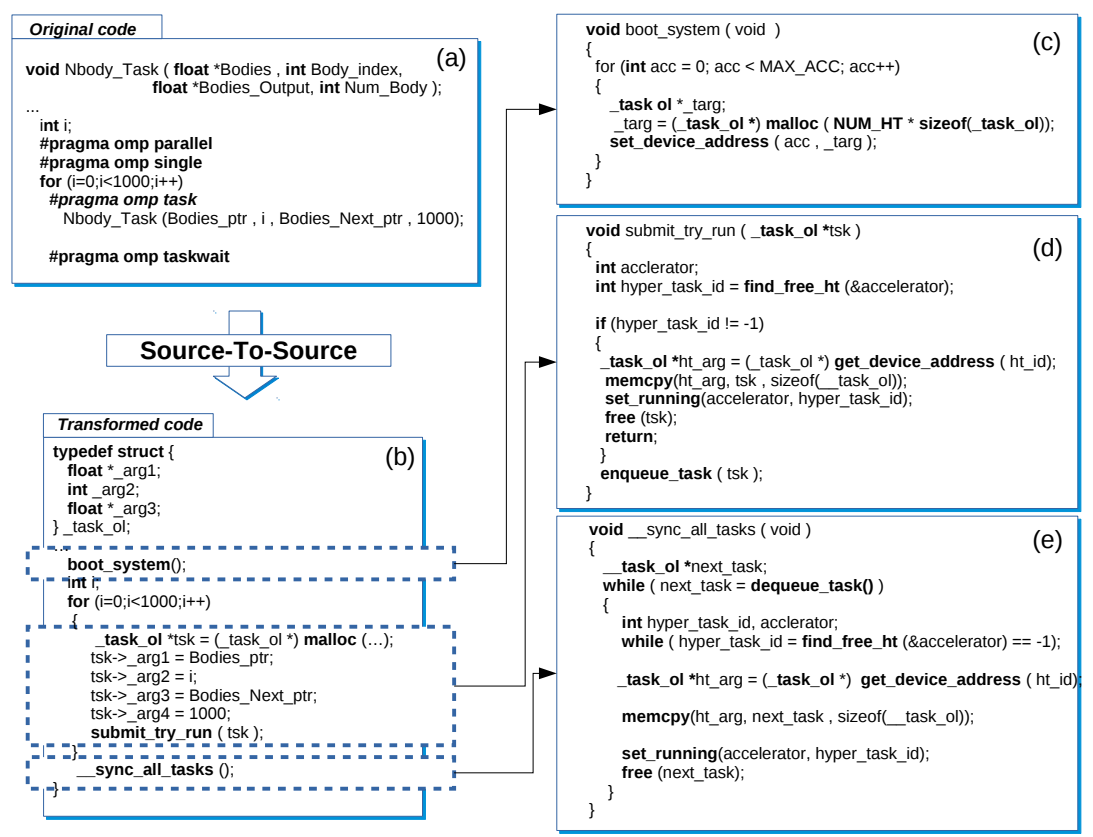

Fig. 4: Source-to-source transformation of a OpenMP task-parallel application (a) into a set of run-time system calls (b): booting a number of thread (c), creating tasks (d) and a task-barrier (e).

a)

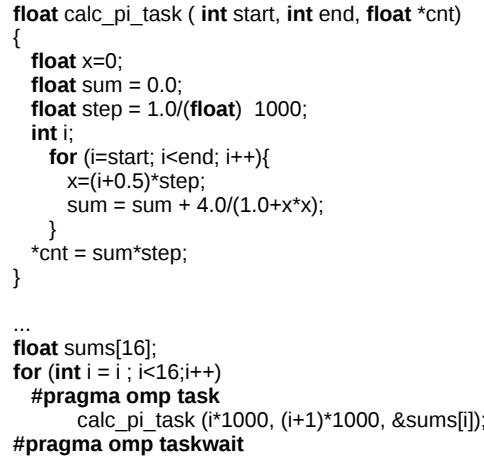

float calc_pi_task ( int start, int end, float ${ }^{*}$ cnt)

float $x=0$

float sum $=0.0$;

float step $=1.0 /$ (float) 1000

int $\mathrm{i}$;

or $(i=s t a r t ; i<e n d ; i++)\{$

$x=(i+0.5)^{\star}$ step;

sum $=$ sum $+4.0 /\left(1.0+x^{\star} x\right)$

float sums[16]

for $($ int $i=i ; i<16 ; i++$

\#pragma omp task

calc pi task $\left(i \star 1000,(i+1)^{\star} 1000, \&\right.$ sums[i] $)$

\#pragma omp taskwait

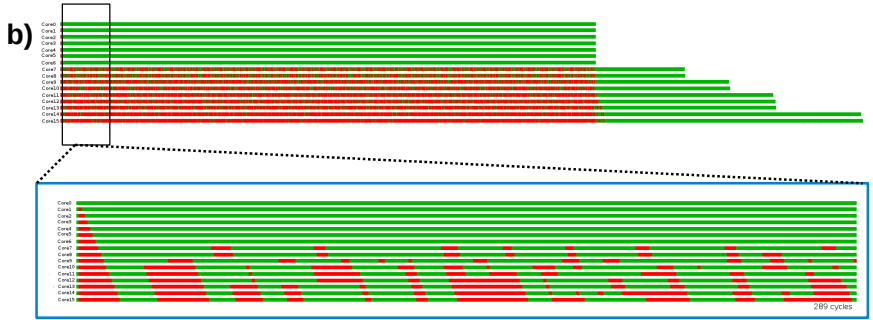

Fig. 5: (a) A simple kernel written with OpenMP directives, where 16 tasks are exposed to concurrently calculate PI. (b) Paraver time-line trace of the kernel execution on an OpenMP accelerator with 16 hyper-tasks, showing blocked (red) and running (green) states of the hyper-tasks.

PI application (Figure 5:a) is compiled down to an accelerator with 16 hyper-tasks. When the application executes, 16 tasks are created to perform the PI-calculation concurrently on the same accelerator but on different hyper-tasks.

Figure 5:b shows the trace generated when executing the tasks concurrently on the OpenMP accelerator. The trace, simulated using Altera Modelsim (with our trace-collection facilities added) and visualized using Paraver [18], shows the state of each hyper-tasks in the accelerators - green color means it is actively executing while red indicates that the hyper-task is blocked. Notice how hyper-tasks with a low identifier execute uninhibitedly (without blocking) while hypertasks with high identifier blocks a lot. This is due to be being blocked awaiting arbitration decisions, and because of the fixed-priority arbitration, hypertasks with a low identifier will more often get permission to regional components while other hyper-tasks are blocked.

\section{REsults}

\section{A. Experimental Setup}

1) System: Evaluation was done using the Altera/Terasic DE5-Net board that contains a Altera Stratix-V GX FPGA (5SGXEA7N2F45C2). The auto-generated system contains the NiosII/f processor, a JTAG-UART interface and a timer, all connected through the Avalon interconnect. Altera Quartus II v14.0 was used for synthesis of the generated systems.

The auto-generated system always generated four banks of on-chip memory to increase the memory bandwidth. The perbank data-width varied between 32- and 512 bits and depends on the vector width used in the OpenMP tasks. The clockfrequency was set according to Altera Timing Analyzer in order to meet all timing requirements (to meet critical path for $80^{\circ} \mathrm{C}$ ). The hardware components in our study were taken from the Altera library and all floating-point components are using single-precision.

We compared our generated OpenMP accelerators with the 1.1 GHz, 57-core Xeon PHI coprocessor and a $2.7 \mathrm{GHz}$ AMD Opteron 48-core SMP (AMD Opteron 6172) system. 
We used the identical input source-code for all benchmarks except the matrix multiplication. For the matrix multiplication, we both used the naive implementation that our compiler compiles with, but we also include the ATLAS (for the Opteron) and Intel MKL (for the Xeon PHI) versions. For the AMD Opteron system, we used the GNU C Compiler (4.9.2) with $-\mathrm{O} 2$ with OpenMP support enabled (-fopenmp). For the Xeon PHI system we used the Intel C Compiler (14.0.2) with -02 and OpenMP support enabled (-openmp). All Xeon PHI experiments were executed natively on the device (no transfer overheads). We hand-optimized our intermediate code to accommodate loop-unrolling that our compiler does not yet have. The hardware generated was the best versions found by the constraint solver after 15 minutes of runtime.

We compared the performance of all three systems by normalizing the performance against the uni-accelerator OpenMP accelerator version:

$$
\text { speedup }=\frac{t_{\text {serial }(A C C)}}{t_{\text {parallel }}}
$$

2) Benchmarks: We used the following kernels to evaluate our OpenMP accelerators:

PI-calculation iteratively approximates the constant $\pi$ by calculating the continued fraction ( 1 billion iterations)

Mandelbrot Fractal draws the Mandelbrot fractal on 4096x4096 pixel-map

MatMul calculates the product of two matrixes $(1024 \times 1024$ w/ block-size: 64x64)

N-Body simulation simulates the Newtonian forces between 16384 heavenly bodies

Raytrace tests for 680 million ray-triangle intersection (based on [15])

\section{B. Results}

The performance of our OpenMP task accelerators are plotted in Figure 6. For the PI-kernel, Figure 6:a, our OpenMP accelerators performs significantly better than both the Xeon PHI and the AMD Opteron on an accelerator to core basis. Performance can reach as high as 20x faster than one AMD Opteron, requiring several AMD Opteron sockets to reach similar performance levels. Xeon PHI performs slightly better than the AMD Opteron but is $17 \%$ slower than our OpenMP accelerators. The PI OpenMP accelerator generated by our model is limited by the amount of DSP-blocks available on the device.

The OpenMP accelerator performs the poorest on the NBody kernel in Figure 6:b. Both the Xeon PHI and the AMD Opteron outperforms the accelerator with almost a magnitude of performance improvements. There are two reasons for this: our OpenMP accelerators lacks caches and since the N-Body kernel is memory-intensive, there is a large contention on the shared bus. The second problem is that our N-Body kernel is not vectorized, while the versions of both AMD Opteron and Xeon PHI are.

The Raytrace benchmark (Figure 6:c) is another case where the OpenMP accelerator perform worse than the AMD Opteron. Surprisingly, the OpenMP accelerators are still on par with the Xeon PHI cores with near equivalent performance. The main limitation for the accelerators is the memory bandwidth, where we found significant time spent blocking on memory reads/writes.

The matrix multiplication case shows how well the OpenMP accelerators can perform, even without caches (Figure 6:d). Here the OpenMP accelerators had 512-bit vector floating point units, exploiting the massive SIMD parallelism available in the multiplications. The performance is on par with both the Intel MKL library on Xeon PHI and ATLAS on AMD Opteron on an accelerator to core basis. The main limitation to performance is the amount of DSP-blocks in our FPGA and the lack of caches. When using the identical source code (marked -identical in the graph), our accelerators outperform the general purpose systems.

The OpenMP accelerators perform on par with the Xeon PHI for the Mandelbrot fractal generator, while the AMD Opteron outperforms both by a factor of 5x. The limiting factor for the OpenMP is the area it occupies on the FPGA. Also, our OpenMP accelerators does not use vectorizing support. Interestingly, the Mandelbrot fractal kernel was the only kernel the constraint programming model deemed worth of having more than eight hyper-tasks per accelerators.

Overall, the automatically generated systems fare well compared to modern ASIC processors. Our OpenMP accelerators executed under a clock frequency of between $90 \mathrm{MHz}-100$ $\mathrm{MHz}$, which is far below the several $\mathrm{GHz}$ that the two other machines used, yet yielded comparable performance.

\section{CONCLUSION AND Discussion}

In this paper, we have shown design direction for an OpenMP driven hardware synthesis. We introduce the hypertasks - instances of the OpenMP task primitive - that reside within accelerators and that can exploit MIMD, SIMD and SPMD capabilities. We introduced regional components components that can be shared within an accelerator across several hyper-tasks. We have shown how to formulate and solve hardware generation through constraint-programming.

We showed that our OpenMP accelerators can provide comparable performance-levels to modern high-end systems running the very same OpenMP application. The methods described are portable - every new generation of FPGAs that are released can support them. As the capacity of future FPGAs grows, so will the performance obtained through our methods. Additionally, our method minimizes the expected input from the user (letting most of the work be performed by the model).

The main limitation is that we do not model the memory hiearchy - there are no per-accelerator caches. This limits the performance, as every memory access will have to go out on the shared bus. While adding a cache would be trivial, it is also counter-intuitive as we would expect the user to provide the type and size for the cache. Our intention for the future is to model the caches and the rest of memory hierarchy.

\section{ACKNOWLEDGEMENTS}

The reviewers, for valuable comments that helped making the paper better. Christian Schulte, Gabriel Hjort Blindell and Roberto Castaneda Lozano, for providing feedback during early stages of the paper. 


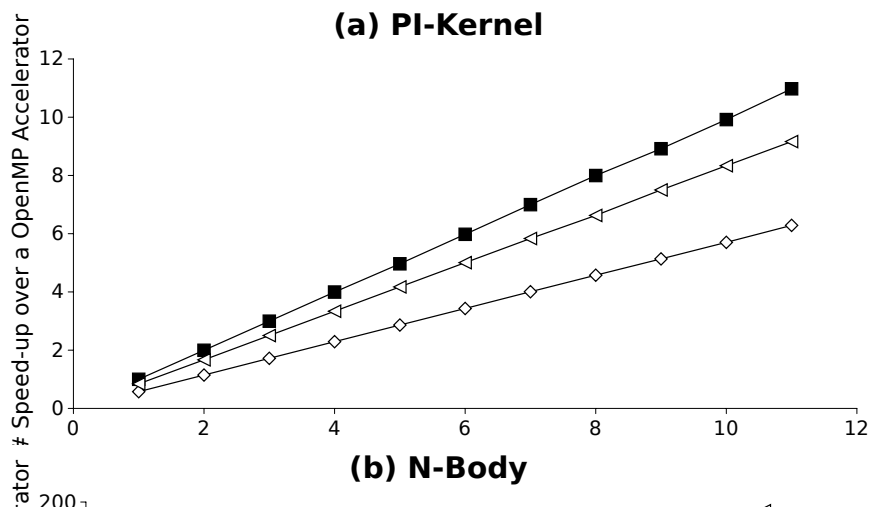

\section{REFERENCES}

[1] Altera. Avalon Interface Specifications, mar 2015.

[2] D. Cabrera, X. Martorell, G. Gaydadjiev, E. Ayguade, and D. JiménezGonzález. OpenMP extensions for FPGA Accelerators. In Systems, Architectures, Modeling, and Simulation, 2009. SAMOS'09. International Symposium on, pages 17-24. IEEE, 2009.

[3] J. Choi, S. Brown, and J. Anderson. From software threads to parallel hardware in high-level synthesis for FPGAs. In Field-Programmable Technology (FPT), 2013 International Conference on, pages 270-277. IEEE, 2013.

[4] J. Cong and W. Jiang. Pattern-based behavior synthesis for FPGA resource reduction. In Proceedings of the 16th international ACM/SIGDA symposium on Field programmable gate arrays, pages 107-116. ACM, 2008.

[5] T. S. Czajkowski, U. Aydonat, D. Denisenko, J. Freeman, M. Kinsner, D. Neto, J. Wong, P. Yiannacouras, and D. P. Singh. From OpenCL to high-performance hardware on FPGAs. In Field Programmable Logic and Applications (FPL), 2012 22nd International Conference on, pages 531-534. IEEE, 2012.

[6] A. Filgueras, E. Gil, D. Jimenez-Gonzalez, C. Alvarez, X. Martorell, J. Langer, J. Noguera, and K. Vissers. OmpSs@Zynq All-programmable SoC Ecosystem. In Proceedings of the 2014 ACM/SIGDA International Symposium on Field-programmable Gate Arrays, FPGA '14, pages 137146, New York, NY, USA, 2014. ACM.

[7] J. Fowers, G. Brown, P. Cooke, and G. Stitt. A performance and energy comparison of FPGAs, GPUs, and multicores for sliding-window applications. In Proceedings of the ACM/SIGDA international symposium on Field Programmable Gate Arrays, pages 47-56. ACM, 2012.

[8] D. Gohringer, M. Birk, Y. Dasse-Tiyo, N. Ruiter, M. Hubner, and J. Becker. Reconfigurable MPSoC versus GPU: Performance, power and energy evaluation. In Industrial Informatics (INDIN), 2011 9th IEEE International Conference on, pages 848-853. IEEE, 2011.

[9] R. J. Halstead and W. Najjar. Compiled multithreaded data paths on FPGAs for dynamic workloads. In Proceedings of the 2013 International Conference on Compilers, Architectures and Synthesis for Embedded Systems, page 3. IEEE Press, 2013.

[10] R. Kalarot and J. Morris. Comparison of FPGA and GPU implementations of real-time stereo vision. In Computer Vision and Pattern Recognition Workshops (CVPRW), 2010 IEEE Computer Society Conference on, pages 9-15. IEEE, 2010.

[11] S. Kestur, J. D. Davis, and O. Williams. BLAS Comparison on FPGA, CPU and GPU. In ISVLSI, pages 288-293, 2010.

[12] Labor and U. D. Bureau of Labor Statistics. Occupational Outlook Handbook 2010-2011. 2010.

[13] Y. Leow, C. Ng, and W.-F. Wong. Generating hardware from OpenMP programs. In Field Programmable Technology, 2006. FPT 2006. IEEE International Conference on, pages 73-80. Ieee, 2006.

[14] K. Martin, C. Wolinski, K. Kuchcinski, A. Floch, and F. Charot. Constraint-driven identification of application specific instructions in the DURASE system. In Embedded Computer Systems: Architectures, Modeling, and Simulation, pages 194-203. Springer, 2009.

[15] T. Möller and B. Trumbore. Fast, minimum storage ray-triangle intersection. Journal of graphics tools, 2(1):21-28, 1997.

[16] F. Nowak, M. Bromberger, and W. Karl. An Architecture Framework for Porting Applications to FPGAs. In Architecture of Computing Systems (ARCS), 2014 27th International Conference on, pages 1-7. VDE, 2014.

[17] A. Papakonstantinou, K. Gururaj, J. A. Stratton, D. Chen, J. Cong, and W.-M. Hwu. FCUDA: Enabling efficient compilation of CUDA kernels onto FPGAs. In Application Specific Processors, 2009. SASP'09. IEEE 7th Symposium on, pages 35-42. IEEE, 2009.

[18] Pillet, Vincent and Labarta, Jesús and Cortes, Toni and Girona, Sergi. Paraver: A tool to visualize and analyze parallel code. In Proceedings of WoTUG-18: Transputer and occam Developments, volume 44, pages $17-31,1995$.

[19] A. Podobas. Accelerating Parallel Computations with OpenMP-Driven System-on-Chip Generation for FPGAs. In Embedded Multicore/Manycore SoCs (MCSoc), 2014 IEEE 8th International Symposium on, pages 149-156. IEEE, 2014.

[20] C. Schulte, M. Lagerkvist, and G. Tack. Gecode. Software download and online material at the website: http://www. gecode. org, 2006.

[21] A. Severance and G. Lemieux. VENICE: A compact vector processor for FPGA applications. In Field-Programmable Technology (FPT), 2012 International Conference on, pages 261-268. IEEE, 2012.

Fig. 6: Performance comparison for the various benchmarks running on our OpenMP accelerators. 